\title{
Tests and Measurements of the CESR Phase III Interaction Region Superconducting Magnets *
}

\author{
J. Welch, G. Codner, G. Dugan, A. Temnykh, Cornell University, Ithaca NY 14850 \\ A. Jain, J. Muratore, BNL, Upton, NY 11973-5000
}

\section{INTRODUCTION}

Superconducting interaction region magnets for the CESR ring have been built by industry and tested. They will allow for higher luminosity by reducing the long range beam-beam effect at the crossing point nearest to the interaction point (IP). They also provide the necessary energy flexibility needed for charm physics operation in the future. The design of these magnets places them as close as physically possible to the IP, immersed within the 1.5 T CLEO detector solenoid field. Consequently the peak beta functions are modest, similar to arc values, and the chromaticity contribution is minimized.

The magnets consist of five identical 'units' each with three independent sets of coils. The main quadrupole (MQ) coils are capable of gradients of up to $48.4 \mathrm{~T} / \mathrm{m}$ at $1225 \mathrm{~A}$. Skew quadrupole (SQ) coils are nested over the MQ coils and generate a field about $10 \%$ as strong. Dipole (D) windings occupy the outermost layer. They can produce a horizontal dipole field up to $0.13 \mathrm{~T}$ for vertical orbit correction.

\section{TESTS AND MEASUREMENTS}

All magnets were quench tested in vertical test cryostats. Units 1 through 4 were assembled into two cryostats - unit 5 remains a spare. Field quality was measured on unit 1 before and after it was placed in the cryostat, otherwise field quality measurements were made on magnets in cryostats.

\subsection{Field Quality}

Harmonic Measurements Integral field harmonics were measured at Cornell and at BNL using two different techniques. At Cornell, we measured the integral field with a $9.5 \mathrm{~mm}$ wide, 3 meter long coil consisting of 4 turns. The coil ends were fixed on rotating stages outside the cryostat. The integral field at each point on a circle of $50 \mathrm{~mm}$ radius was measured by flipping the coil by 180 degrees. A Fourier analysis was used to obtain the multipoles, which were evaluated at a reference radius of $50 \mathrm{~mm}$.

The integral harmonics in the warm state were measured at BNL in units 1 and 5 using a rotating coil system of five $0.92 \mathrm{~m} \mathrm{long}^{1}, 74 \mathrm{~mm}$ radius tangential windings. This system allowed very precise measurements of the field harmonics even for warm measurements carried out at $1 \mathrm{~A}$ for

* Work at Cornell was supported by the National Science Foundation. The tests at BNL were supported by the US Department of Energy under contract No. DE-AC02-98CH10886

${ }^{1}$ Since the length of the measuring coil in the BNL measurements was only $0.92 \mathrm{~m}$, measurements of integral field quality were also carried out by adding data from two adjacent positions of the measuring coil, covering a total length of $1.84 \mathrm{~m}$. No significant differences between $0.92 \mathrm{~m}$ coverage and $1.84 \mathrm{~m}$ coverage were seen, implying that the length of 0.92 $\mathrm{m}$ was adequate.
Table 1: Integral field quality of the MQ's. Harmonics are given in "units" at $50 \mathrm{~mm}$ radius, normalized to 10,000 units of the MQ field.

\begin{tabular}{|c|c|c|c|c|c|c|c|}
\hline & \multicolumn{3}{|c|}{ \#1 } & \#2 & \#3 & \#4 & \#5 \\
\hline & $\mathrm{CU}$ & $\mathrm{BNL}^{\mathrm{I}}$ & BNL & $\mathrm{CU}$ & $\mathrm{CU}$ & $\mathrm{CU}$ & $\mathrm{BNL}^{1}$ \\
\hline b2 & 7.0 & -3.66 & -3.90 & -29.4 & -6.8 & 5.7 & -10.14 \\
\hline b3 & 1.6 & 0.88 & 0.99 & -4.6 & -3.4 & -5.4 & -4.28 \\
\hline b4 & 0.1 & -0.34 & -0.11 & -0.9 & -0.9 & 0.9 & -1.87 \\
\hline b5 & -8.5 & -6.93 & -7.73 & -7.6 & -7.5 & -6.1 & -6.45 \\
\hline b6 & 0.0 & 0.07 & -0.05 & -0.5 & 0.4 & -0.3 & -0.37 \\
\hline b7 & 0.0 & -0.07 & -0.11 & 0.2 & 0.1 & 0.3 & -0.25 \\
\hline b8 & -0.1 & -0.03 & -0.05 & -0.1 & -0.3 & 0.0 & -0.08 \\
\hline b9 & -0.6 & -0.46 & -0.44 & -0.5 & -0.7 & -0.6 & -0.54 \\
\hline $\mathrm{a} 2$ & 1.6 & -2.25 & -2.65 & 4.8 & 2.1 & -20.6 & 1.83 \\
\hline a3 & 2.2 & 0.56 & 0.04 & -0.1 & -0.4 & 2.2 & -0.65 \\
\hline $\mathrm{a} 4$ & -1.4 & -1.18 & -1.00 & 0.4 & 0.5 & 1.5 & -0.52 \\
\hline a5 & 1.9 & -0.42 & -0.39 & 1.3 & 1.7 & 0.7 & -0.77 \\
\hline a6 & 0.3 & -0.18 & -0.21 & 0.4 & 0.4 & 0.6 & 0.12 \\
\hline a7 & 0.6 & -0.29 & -0.38 & 0.2 & 0.5 & 1.1 & 0.13 \\
\hline a8 & 0.5 & 0.01 & 0.03 & 0.2 & 0.3 & 0.4 & 0.00 \\
\hline a9 & 0.3 & 0.00 & 0.00 & 0.3 & 0.2 & 0.4 & -0.02 \\
\hline
\end{tabular}

${ }^{1}$ Warm measurement

the MQ and $0.2 \mathrm{~A}$ for the D and the SQ. Measurements were carried out for both polarities of the current and any remnant fields were subtracted out by using the two sets of data.

The results of the integral measurements at Cornell and BNL are summarized in Table 1 for the MQ. The integral transfer function measured by the same rotating coil at BNL showed an $\approx 0.5 \%$ increase on cool down and had a value of $26.14 \mathrm{~T} / \mathrm{kA}$ at $1200 \mathrm{~A}$ in unit 1 . The field harmonics measured cold were in excellent agreement with the warm measurements. The Cornell and BNL results are in reasonable agreement, except for the sextupole terms. This discrepancy may be due to the fact that the Cornell measurements were carried out with the magnets inside a cryostat, and may have been influenced by magnetic material nearby

The integral transfer function in the SQ was measured to be $\approx 10 \mathrm{~T} / \mathrm{kA}$. The most dominant harmonic was found to be several units of b2. Similarly, in the skew dipole layers, the integral transfer function is $0.41 \mathrm{Tm} / \mathrm{kA}$ and the dominant error term is the skew sextupole at the level of $\approx 1.3 \%$ of the dipole field.

Z-Scans of MQ Field Quality A striking result seen from Table 1 is the large normal sextupole, b2, in unit 2 and large skew sextupole, a2, in unit 4 . The magnitude of these harmonics is larger than expected from construction 


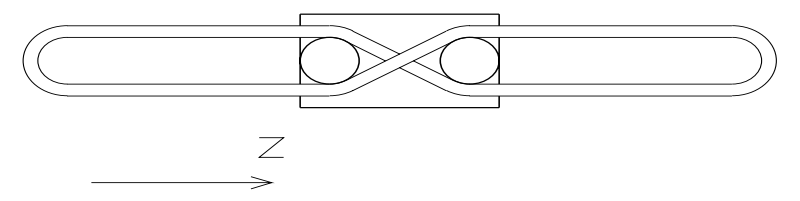

Figure 1: Coil used for Z-scan at Cornell

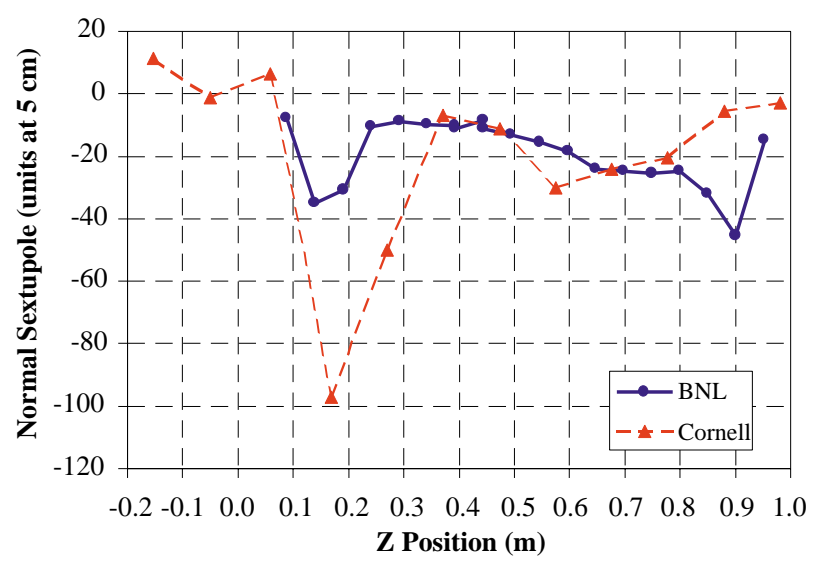

Figure 2: Sextupole distribution measured along the magnet axis.

tolerances. This motivated us to explore the axial distribution of the multipole components in these magnets. These measurements were carried out both at Cornell and at BNL using a slight variation of the integral measurements described earlier. In the Cornell measurements, the flip coil has been modified as shown in Fig. 1. One end of the coil was rotated by 180 degrees and a small "slider" was inserted at the crossover region. The multipole distribution along $z, b_{n}(z)$ was found by differentiating the measured signal by the "slider" position. The slider was moved in steps of $10 \mathrm{~cm}$ for these measurements. Since the slider position along the Z-axis was not well defined, the estimated precision of these measurements is around $10 \%$.

In the Z-scans at BNL, a coil of 0.91 m length and 38 $\mathrm{mm}$ radius was used. A series of measurements were carried out by moving the coil in $\approx 50 \mathrm{~mm}$ steps. The field harmonics in any $50 \mathrm{~mm}$ region were then obtained by subtracting the corresponding measurement from the adjacent one. Due to subtraction of nearly equal signals, the results have a relatively larger error of 1 unit. The results of the Zscans in Unit 2 are shown in Fig. 2 for the normal sextupole component of unit 2 .

MQ Field Quality Discussion All five MQ's have approximately -7 units of the first allowed nonlinear component b5 with a variation from magnet to magnet of about 1 unit. The design value for b5 is about -0.45 units. The difference is almost certainly due to systematic construction errors $^{2}$. The most likely candidates are the spacer shapes which tended to be too thick at the inner radius, and geometric distortions in the coil ends. The fact that there is so little variation in the $b 5$ terms from magnet to magnet attest

\footnotetext{
${ }^{2}$ Calculations show that even with extreme assumptions for the permeability of the stainless steel central post, b5 would not change more than 1 unit.
}

to the care with which the coils were wound and shimmed. The specification was that the sum of all harmonics be less than 5 units.

Many possibilities were investigated and ruled out for the origin of the anomalous sextupole fields: magnetic permeability of welds - insufficient heat affected zone even if fully magnetize, inconsistent with z-scan pattern; turn-toturn shorts - in the cold state the superconductor should determine the current path but there was little difference between cold and warm measurements, there were no quench problems which might have been expected if current was being conducted through the copper portion of the wire; fields generated by nearby steel used to support the cryostat - measurements were checked at BNL on a granite table; various possible measurement errors - similarity of the measurements at BNL and Cornell using different techniques and coils.

Model of Geometric Errors The only tenable hypothesis was that the geometry of the assembled coils was distorted. A simple two-dimensional model of the quadrupole was made, in which the three blocks comprising each coil were replaced with a single block of uniform current density. The entire quadrupole was modeled as eight such blocks. Input variables were the widths and centroids of the azimuthal gaps between the blocks, and the radial location of each block.

Assuming no radial errors, the widths and centroids of the gaps necessary to produce the measured normal and skew harmonics (from sextupole through duodecapole) were found by a fitting procedure. This was done as a function of longitudinal position along the length of the quadrupole. For both unit 2 and unit 3 , the fitted gap widths were comparable with those obtained from direct mechanical measurements made on the coils during assembly. However, for unit 2 the fitted gap centroid errors ranged up to $0.6 \mathrm{~mm}$ near one end of the quadrupole larger than what was measured during assembly. Similarly, if one assumes no azimuthal coil errors, the fitted radial coil errors ranged up to $0.4 \mathrm{~mm}$ - larger than expected from construction tolerances $(0.1-0.2 \mathrm{~mm})$. With correlated combinations of radial and azimuthal errors, the sizes of the fitted errors needed to explain the sextupole harmonic were reduced, but are still somewhat larger than could be explained by mechanical measurements and tolerances.

\subsection{Quench and Cryogenics Behavior}

Unit 1 was subjected to the most extensive quench testing, including testing atTesla Engineering, BNL and Cornell. Quench tests were done at $4.5 \mathrm{~K}$ to check the mechanical performance of the magnet assembly under its operating fields and consisted of ramping a layer either alone or with other layers powered until a quench occurred. Each layer was powered independently by its own power supply. Ramp rates at BNL were $3 \mathrm{~A} / \mathrm{s}$ for the MQ and $5 \mathrm{~A} / \mathrm{s}$ for the SQ and the D.

Fig. 3, quenches 1-21, shows the performance of the MQ alone and demonstrates gradual but steady training to and past the maximum operating current of $1225 \mathrm{~A}$. Quenches occurred in different locations and did not settle into any 


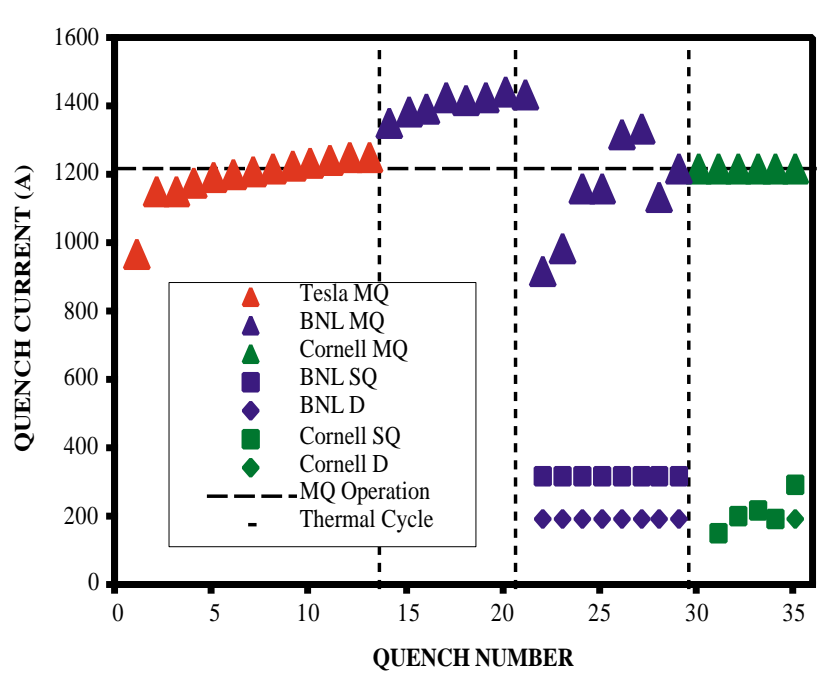

Figure 3: Unit 1 quench training history.

one quadrant, and this implies that the MQ was still training at $1440 \mathrm{~A}$, about $89 \%$ of the theoretical conductor limit. Note that the training is not reversed by putting the magnet through thermal cycles. The test at Tesla was of the MQ alone before the other layers had been added. The test at BNL, quenches 16-29, took place after the other layers had been added and the shrink rings had been re-installed. Note the $100 \mathrm{~A}$ increase in quench current. After performance was established for the MQ, further quench testing was done to show the effect of the other layers. At BNL, this was done by powering the SQ and the D at their maximum operating currents of $325 \mathrm{~A}$ and $200 \mathrm{~A}$, respectively, and ramping the main quadrupole at $3 \mathrm{~A} / \mathrm{s}$ until a quench occurred in the SQ or D, as shown by quenches 22-29. The MQ current increased to $1345 \mathrm{~A}$ at the fifth quench, (in which the MQ also quenched), then decreased to $1142 \mathrm{~A}$ with no quench in the MQ. After this, numerous power cycles were done which demonstrated stable operation of the three layers at their maximum operating currents.

Additional quench tests were done at Cornell after electro-mechanical work was finished and the magnet was inserted into its cryostat. In this case, the MQ was ramped to $1225 \mathrm{~A}$ and the other layers were ramped until a layer quenched. As can be seen in Fig. 3 quenches 31-36, both the SQ and the D layers trained back to their respective maximum operating currents of $325 \mathrm{~A}$ and $200 \mathrm{~A}$.

Solenoid Test The magnet was installed with its nonlead end (bottom in the vertical test dewar) inserted into a solenoid magnet built at Cornell and designed to provide a $1.5 \mathrm{~T}$ field at $1000 \mathrm{~A}$ to simulate the field in the CLEO detector. For this test, the MQ was ramped to $1225 \mathrm{~A}$ and then the solenoid was ramped at $5 \mathrm{~A} / \mathrm{s}$ to $1000 \mathrm{~A}$. The MQ quenched when the solenoid reached $795 \mathrm{~A}$, then $896 \mathrm{~A}$ during a second ramp. After this, the solenoid power supply started oscillating and the test had to be terminated. These results imply that the MQ was training under the forces imposed by the solenoidal field.

A complication to quench protection resulted from magnetic coupling of the nested coils. Although there was no mutual inductance between any two sets of windings, there was substantial magnetic flux shared between the D and pairs of SQ and MQ coils. Since the quench system compared voltages from pairs of coils, (the sign of the comparison was such that the difference voltage during current ramping is zero) a rapid change in dipole current induced voltages in an opposing pair SQ coils which was seen as apparent SQ quench. This unrecognized coupling had apparently been the cause of some anomalous quench behavior both at TESLA and at BNL.

Cryogenics Performance During the initial cooldown of cryostat 1 at Cornell there were two events which generated sudden internal motion that was observed and heard outside the cryostat. A series of G10 spacers that guide the LN shield in the 'neck' region of the cryostat is suspected. There were instrumentation problems leading to erroneous helium level measurements and overfilling. Once these were overcome the heat leak was measured at approximately $16 \mathrm{~W}$ liquifaction and $0.3 \mathrm{~g} / \mathrm{s}$ refrigeration load. Loose bolts opened up a leak during a quench but the seal was re-established after partial warm-up and tightening the bolts. Some small flanges were found to have vacuum leaks which were repaired after warm up. The AMI vapor cooled leads all performed within the manufacturers specifications.

\section{SUMMARY}

Five interaction region magnets have been quench tested and their field quality measured. The quench performance is uniformly good, within specification with little or no retraining upon thermal cycle. The b5 component of the MQ coils was systematically outside specification but within tolerable levels. Two magnets exhibited large sextupole or skew sextupole MQ components probably due to geometric distortion of the coil assemblies. The ultimate cause of these distortion has not been determined.

\section{ACKNOWLEDGEMENTS}

We would like to acknowledge the conscientious and careful work done at Tesla Engineering where the magnets were fabricated, especially the efforts of D. Coxill, D. Langrebe and J. Coughlin. Special thanks also go to A. Ijspeert of CERN for giving expert guidance over a long period of time, and the staff and management at magnet measurement group at BNL for supporting the measurements of the Cornell magnets.

\section{REFERENCES}

[1] J.J.Welch, G.F. Dugan, E. Nordberg, D. Rice, Cornell University, The Superconducting Interaction Region Magnet System for the CESR Phase III Upgrade, PAC99 